\title{
Prenatal choline availability alters the context sensitivity of Pavlovian conditioning in adult rats
}

\author{
Jeffrey A. Lamoureux, Warren H. Meck, ${ }^{1}$ and Christina L. Williams \\ Department of Psychology and Neuroscience, Duke University, Durham, North Carolina 27708, USA
}

\begin{abstract}
The effects of prenatal choline availability on Pavlovian conditioning were assessed in adult male rats (3-4 mo). Neither supplementation nor deprivation of prenatal choline affected the acquisition and extinction of simple Pavlovian conditioned excitation, or the acquisition and retardation of conditioned inhibition. However, prenatal choline availability significantly altered the contextual control of these learned behaviors. Both control and choline-deprived rats exhibited context specificity of conditioned excitation as exhibited by a loss in responding when tested in an alternate context after conditioning; in contrast, choline-supplemented rats showed no such effect. When switched to a different context following extinction, however, both choline-supplemented and control rats showed substantial contextual control of responding, whereas choline-deficient rats did not. These data support the view that configural associations that rely on hippocampal function are selectively sensitive to prenatal manipulations of dietary choline during prenatal development.
\end{abstract}

There is increasing evidence that variations in maternal dietary choline intake during the second half of pregnancy cause structural, biochemical, and physiological changes in basal forebrain neurons and their projections to the hippocampal complex as well as long-term cognitive changes in the offspring (e.g., Meck and Williams 2003; McCann et al. 2006; Meck et al. 2008). We know, for instance, that the adult offspring of pregnant rats supplemented with 4.5 times the amount of choline in the standard laboratory diet display improved memory capacity and precision on the radial-arm maze (e.g., Meck et al. 1988, 1989; Meck and Williams 1997b, 1999; Tees 1999a), Morris water maze (e.g., Tees 1999b; Tees and Mohammadi 1999; Yang et al. 2000; Brandner 2002), as well as facilitation of sustained attention and interval timing (e.g., Meck and Williams 1997a,c; Mohler et al. 2001; Cheng et al. 2006, 2008a,b; Cheng and Meck 2007) compared with offspring of dams fed a standard diet. Choline deficiency during the same developmental time frame, embryonic days (ED) $12-17$, results in impaired performance on some, but not all, of these behavioral measures (e.g., Meck and Williams 1999, 2003). Furthermore, perinatal choline supplementation can alter behavior following a variety of developmental disorders, including the alleviation of abnormalities associated with fetal alcohol syndrome in rats (Thomas et al. 2000, 2004, 2007; Wagner and Hunt 2006), attenuation of some of the motor deficits observed in a Mecp $21^{\text {lox }}$ mouse model of Rett syndrome (Nag and BergerSweeney 2007), and the improvement of sensory gating in a DBA/2 mouse model of schizophrenia that exhibits reduced numbers of hippocampal a7 nicotinic receptors (Stevens et al. 2008).

These choline-induced alterations in cognitive function are accompanied by changes in the size and shape of basal forebrain cholinergic neurons (e.g., Williams et al. 1998; McKeon-O'Malley et al. 2003); modifications in acetylcholine turnover and choline transporter expression in the septum and hippocampus (Cermak et al. 1999; Mellott et al. 2007b); modulation of hippocampal neurogenesis, gene expression, phospholipase D activity, NGF levels, and MAPK and CREB activation (e.g., Holler et al. 1996; Sandstrom et al. 2002; Mellott et al. 2004, 2007a; Glenn et al. 2007); changes in dendritic fields and spine density in CA1 and

\footnotetext{
'Corresponding author.
}

E-mail meck@psych.duke.edu; fax (919) 660-5798.

Article is online at http://www.learnmem.org/cgi/doi/10.1101/lm.1058708. dentate gyrus (DG) regions of the hippocampus (Meck et al. 2008); as well as modification of the neuropathological response to status epilepticus (e.g., Holmes et al. 2002; Wong-Goodrich et al. 2008a) and thresholds for eliciting long-term potentiation (LTP) in the hippocampus (Pyapali et al. 1998; Jones III et al. 1999). Together, these findings suggest that alterations in choline availability during early development may have specific impact on the ontogeny and later functioning of basal forebrain cholinergic neurons as well as efferent neurons involved in hippocampal LTP (Montoya et al. 2000). These findings also predict that behaviors that rely on the hippocampus are likely to be most affected by this dietary manipulation.

Although choline is well known as the precursor for the neurotransmitter acetylcholine, it may be especially crucial to young or developing mammals for a number of other reasons (see Blusztajn and Wurtman 1983; Blusztajn 1998; Zeisel 2000, 2004, 2005). It is the precursor of certain phospholipids (e.g., phosphatidylcholine, sphingomyelin, and plasmenylcholine), which constitute the bulk of phospholipids in all biological membranes. Thus, there may be a particularly high demand for choline during prenatal and neonatal periods associated with rapid neurogenesis and synaptogenesis. Choline can also be enzymatically oxidized to betaine (mostly in peripheral tissues) and the methyl groups of betaine can then be used to resynthesize methionine from homocysteine. Changes in methionine availability alter the methylation of regulatory sequences of genes and of histones, leading to alterations in the patterns of gene expression (e.g., Waterland and Michels 2007; Nafee et al. 2008). Choline is also the precursor of two signaling molecules, plateletactivating factor, and sphingosylphosphorylcholine. Changes in choline availability may also alter membrane synthesis, methylation, and signaling broadly throughout the brain and periphery as well as more restricted effects on cholinergic neuronal pathways (e.g., Zeisel and Blusztajn 1994; Meck and Williams 2003).

One common distinction in the Pavlovian-conditioning literature is between tasks that are sensitive to manipulation of the hippocampal formation from those that are not (e.g., Ross et al. 1984; Meck 1988; Schmajuk and Buhusi 1997; Holland et al. 1999). For example, simple excitatory Pavlovian conditioning is typically found to be unaffected by lesions of the hippocampus, while conditional discriminations in which animals must rely on combinations of predictive cues to respond correctly are disrupted by hippocampal damage (e.g., Jarrard and Davidson 1990, 1991). If prenatal choline availability is altering the development 
of cholinergic neurons in the basal forebrain that project to the hippocampus (see Meck and Williams 2003), our dietary manipulation might only be expected to affect conditioning tasks that require hippocampal involvement, not relatively simple tasks such as excitatory conditioning which do not rely on the hippocampus (e.g., Green and Woodruff-Pak 2000).

In the current series of experiments, we examined the effects of prenatal choline supplementation and deficiency using a series of appetitive Pavlovian-conditioning tasks, all of which require associative learning. Our rationale was to determine whether variations in choline availability during prenatal development altered the learning of a simple association between the conditioned (CS) and unconditioned (US) stimuli (e.g., noise $\rightarrow$ food sequence), or if the dietary manipulation primarily affected conditioning tasks that require more complex relational processing and intact septal-hippocampal function (e.g., context $\mathrm{A}=$ tone $\rightarrow$ food; context $\mathrm{B}=$ noise $\rightarrow$ no food).

In order to assess the importance of prenatal choline availability on associative learning, we investigated basic aspects of appetitive Pavlovian conditioning, i.e., conditioned excitation and extinction (e.g., Pavlov 1927) in experiment 1. In this paradigm, rats first receive repeated trials in which the CS occurs just before the presentation of the US, i.e., in a noise $\rightarrow$ food sequence. During this initial phase of training, the rat develops an increasing tendency to perform the conditioned response (CR) in the presence of the CS indicating that it expects the occurrence of the US. Typically, the probability of the CR increases in a negatively accelerating fashion until it reaches an asymptotic level. If the CS is then repeatedly presented in the absence of the reinforcing US (i.e., noise $\rightarrow$ no food), then the CR gradually declines; this is referred to as extinction of the CR (Gallistel and Gibbon 2000).

One behavioral phenomenon that has been shown to be sensitive to hippocampal manipulation is the discriminative use of contextual cues to control conditioned responding (e.g., Holland and Bouton 1999). Typically, when CS-US pairings occur in one training environment or context, there is a small loss of responding to the CS if it is subsequently presented to the animal in the presence of a different set of contextual cues (e.g., Lovibond et al. 1984; Hall and Honey 1990; Honey et al. 1990; Kaye and Mackintosh 1990). However, this typical decrement in responding with a context switch is not observed in rats with electrolytic or aspiration lesions of the hippocampus (e.g., Good et al. 1997).

In order to assess the effects of prenatal choline availability on contextual control of conditioned responding, we employed a renewal design (e.g., Bouton and Bolles 1979) in experiment 2. In this design, rats receive conditioning in one physical context (context A) prior to extinction in either the same context or a context different from that in which they received the initial CS-US pairings (context B). Finally, all of the rats are retested in the original conditioning context (i.e., context A). Bouton and colleagues (e.g., Bouton and Bolles 1979; Frohardt et al. 2000) have found that when rats that are conditioned in context $\mathrm{A}$ followed by extinction training in context $\mathrm{B}$ are later returned to the original training context for the final testing phase, they show a substantial recovery of the initial CR. Presumably, stimuli contained within the original training context act as reminder cues in this ABA condition, retrieving the memory for the initial acquisition phase (A) of the experiment during the final test phase as opposed to the more recent extinction phase (B). Rats in the AAA condition have no effective cues to discriminate the different phases of the experiment and as a consequence cannot selectively retrieve a specific memory from the sequence. In contrast, test session responding for the ABA condition should be more similar to the low levels observed at the end of the initial extinction phase due to the availability of differential contextual cues. This renewal design is particularly useful in that it provides for the potential to observe treatment effects in both the extinction and the renewal test phases of the experiment. Specifically, either the loss of responding with a context switch during extinction or the response recovery in the renewal test (or both) may be affected by prenatal choline availability. More importantly, those two effects may be due to either the same mechanism (e.g., processing of contextual stimuli) or two different mechanisms (e.g., context conditioning and memory retrieval)potentially resulting in nonlinear effects of prenatal choline availability across the two experimental phases.

A second basic type of associative learning, conditioned inhibition, in which the animal learns to predict the absence of an important event, was described by Pavlov (1927). A typical conditioned-inhibition task consists of training with two types of intermixed trials: On reinforced trials, one CS is followed by reinforcement (e.g., noise $\rightarrow$ food). On other trials, the same CS is paired with a second stimulus in the absence of the reinforcement (i.e., light/noise $\rightarrow$ no food). It is presumed that under these training conditions animals learn that the noise predicts the occurrence of the food, while light, the "conditioned inhibitor," comes to predict the absence of food. That is, light "inhibits" the learned response to noise alone.

A relatively small number of studies have examined the neural substrates of inhibitory learning. Aspiration lesions of the hippocampus, for example, impaired a relatively complex phenomenon called "blocking" of excitatory conditioning, but not the learning of conditioned inhibition (e.g., Solomon 1977; Chan et al. 2001). These data suggest that the hippocampal complex is not required for learning conditioned inhibition. Thus, in order to further assess whether prenatal choline availability affects basic associative learning, experiment 3 was designed to evaluate conditioned inhibition in supplemented (SUP), deficient (DEF), and control (CON) rats. In this experiment, rats were given randomly mixed presentations of reinforced and nonreinforced trial types. As training proceeds, the rats should learn to respond more on reinforced trials than on nonreinforced trials. After acquisition of the discrimination, the rats were presented with a retardation test phase in which the inhibitory light CS was paired with food. Rescorla (1969) described this retardation test as one of the critical measures of conditioned inhibition. Presumably, if the CS is a true inhibitor and predicts the absence of reinforcement at the outset of the retardation test, then acquisition of conditioned responding to the cue should be relatively slow during this phase of testing. Consequently, tests of conditioned inhibition should distinguish among prenatal choline treatment groups if inhibitory mechanisms are strengthened or weakened by prenatal choline availability.

\section{Results}

\section{Experiment 1: Acquisition and extinction of Pavlovian conditioned excitation}

The mean $( \pm$ SEM) responding of all treatment groups during the initial excitatory conditioning sessions is depicted in Figure 1. Two rats, one from the CON group and one from the DEF group, failed to show any appreciable conditioned behavior $(>10 \%$ of time in food cup) and were excluded from data analysis. All treatment groups showed a gradual increase in conditioned responding over the course of the training sessions. A treatment $x$ session ANOVA revealed an overall significant main effect of session $\left(F_{(9,765)}=72.76 ; P<0.01\right)$. However, neither the main effect of treatment nor the treatment $\times$ session interaction reached significance $(F s<1)$, suggesting that neither the rate of 


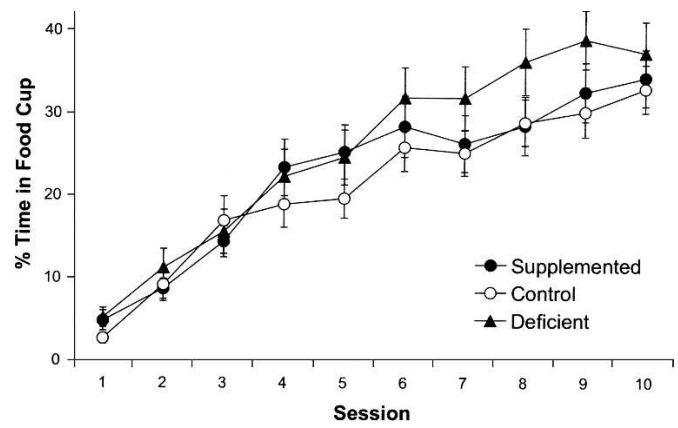

Figure 1. Acquisition of Pavlovian conditioned responding by rats given prenatal choline supplemented (SUP), deficient (DEF), or sufficient $(\mathrm{CON})$ diets. Data shown are session means \pm SEM for the duration of time spent in the food cup during CS presentations on [noise $\rightarrow$ food] training trials.

acquisition nor the asymptotic level of conditioning was affected by prenatal choline availability. These claims were further supported by planned comparisons of the performance of the treatment groups at asymptote; none of the groups differed statistically from one another (largest $F_{(1,85)}=1.57 ; P>0.1$ ). Finally, the shapes of the acquisition curves were compared using trend analysis. The acquisition functions for all treatment groups contained reliable linear and quadratic trends $\left(F \mathrm{~s}_{(1,85)}=171.25\right.$ and 15.48; $P$ s $<0.05$, respectively). However, no group differences were observed in the extent of the polynomial trends, $F \mathrm{~s}<1$. This analysis also supports the claim that the rates of acquisition of conditioning (i.e., the slopes of the learning curves) were similar for the three treatment groups.

The food-cup entries of rats during the four extinction sessions are plotted as a function of prenatal choline availability in Figure 2. The data shown represent conditioned behavior as a percentage of asymptotic responding (i.e., the mean response for each rat during sessions 7-10). Conditioned responding extinguished gradually across the four extinction sessions in all groups of rats. Furthermore, the rate of extinction was not affected by prenatal choline availability. These claims are supported by a treatment $\times$ session ANOVA, which revealed only a reliable main effect of session $\left(F_{(3,81)}=46.93 ; P<0.01\right)$. Neither the main effect of treatment nor the treatment $\times$ session interaction reached significance $(F s<1)$. These claims are further supported by planned pairwise comparisons between treatment groups that revealed no significant differences between any two groups on response rates in the first or last extinction sessions (largest $\left.F_{(1,27)}=1.83 ; P>0.1\right)$. Finally, although the shape of the overall

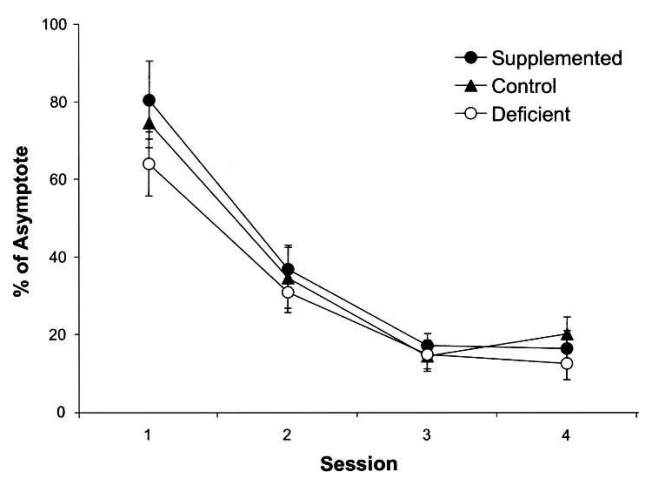

Figure 2. Extinction of Pavlovian conditioned excitation as a function of prenatal choline availability. Data represent session means \pm SEM for responding during the CS on [noise $\rightarrow$ no food] extinction trials, expressed as a percentage of each rat's asymptotic level of responding. response function contained significant linear and quadratic polynomial trends, these trends were not significantly different among the three treatment groups, $F \mathrm{~s}<1$.

The results of experiment 1 suggest that the associative learning abilities underlying the acquisition and extinction of simple Pavlovian conditioned excitation are not affected by the variations in prenatal choline availability. Not surprisingly, this form of Pavlovian conditioning is typically found to be unaffected by lesions of the hippocampus (Holland and Bouton 1999). In contrast, other more complex associative learning tasks in which animals must rely on combinations of predictive cues to respond correctly are well known to be disrupted by hippocampal damage (e.g., Jarrard and Davidson 1990, 1991). If prenatal choline availability is altering the development of specific neuronal populations such as the cholinergic neurons in the basal forebrain that project to the hippocampus (Meck et al. 1989; Williams et al. 1998), our dietary choline manipulation might only be expected to affect relatively complex conditioning tasks that require hippocampal involvement, not relatively simple tasks such as excitatory conditioning.

\section{Experiment 2: Contextual control of Pavlovian conditioned excitation}

During the acquisition phase, rats gradually increased responding to the CS over the course of training. Because two rats, one from the CON group and one from the DEF group, failed to show any appreciable conditioned behavior during acquisition $(>10 \%$ of time in food cup), their data were excluded from further analysis. A treatment $\times$ session ANOVA conducted on the percentage of time rat's spent in the food cup during the CS revealed a significant main effect of session $\left(F_{(9,495)}=48.73 ; P<0.01\right)$, but neither the main effect of treatment nor the two-way interaction of treatment $\times$ sessions reached significance, $F \mathrm{~s}<1$. The different treatment groups also reached a similar level of asymptotic responding. Group means \pm SEM for duration in the food cup on the last session of training were $32.04 \mathrm{~s} \pm 3.96 \mathrm{~s}, 34.51$ $\mathrm{s} \pm 4.29 \mathrm{~s}$, and $36.18 \mathrm{~s} \pm 3.23 \mathrm{~s}$ for the SUP, DEF, and CON treatment groups, respectively. Pairwise comparisons of the asymptotic response levels yielded no significant differences, Fs $<1$.

The mean \pm SEM levels of food-cup entries (expressed as a percentage of asymptotic performance) for each choline treatment group during extinction sessions in the same context (AAA) or in a different context (ABA) are shown in Figure 3 . The responding of all groups reliably decreased over training sessions $\left(F_{(3,159)}=81.17 ; P<0.01\right)$. Also, the majority of rats undergoing extinction in context $\mathrm{B}$ showed less overall responding than those that received extinction training in the context $A$ $\left(F_{(1,53)}=7.27 ; P<0.05\right)$. However, this effect was not observed in all choline treatment groups; i.e., SUP rats did not show lower responding when switched to context B for extinction. These claims were supported by planned contrasts showing that while $\mathrm{DEF}$ and $\mathrm{CON}$ rats responded less in context $\mathrm{B}$ than in context $\mathrm{A}$ $\left(F_{(1,18)}=4.94\right.$, and $F_{(1,17)}=7.41 ; P \mathrm{~s}<0.05$, respectively $)$, this was not true of the SUP rats, $F<1$.

Mean \pm SEM levels of responding during the final test phase in which the CS was presented in context A for all choline treatment groups is shown in Figure 4. Renewal of conditioned responding was observed for both the SUP and CON rats in the ABA condition, but not in the SUP and CON rats that remained in the same context A throughout the entire study (AAA condition) $\left(F_{(1,18)}=16.90\right.$ and $8.49 ; P s<0.05$, respectively). Conversely, this effect was not observed in the DEF rats; DEF rats in both the AAA and the ABA conditions responded at very low levels during the renewal phase of training, $F<1$. 

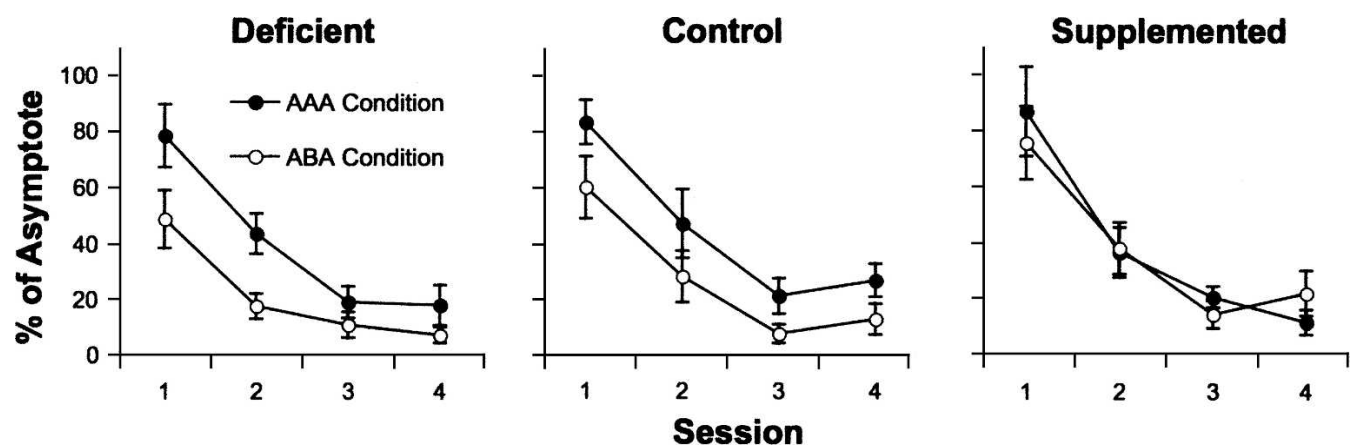

Figure 3. Conditioned responding of rats receiving extinction training in one of two contexts as a function of prenatal choline availability. Data represent group means \pm SEM of the time spent in the food cup during CS presentations, expressed as a percentage of asymptotic responding, for each session of extinction. Rats in the AAA condition received extinction trials in the same training context in which conditioning had occurred. Rats in the $\mathrm{ABA}$ condition received extinction training in an alternate context.

In summary, prenatal choline supplementation and deficiency produced two different effects on the contextual control of Pavlovian conditioning in this experiment. Rats from all choline treatment groups showed a typical decline in responding during extinction; however, both DEF and CON rats showed less overall responding when the CS was extinguished in a novel context (B) rather than the familiar context (A). These results replicate the findings of prior appetitive conditioning studies that examined adult rats bred and raised on standard laboratory chow (e.g., Lovibond et al. 1984; Honey et al. 1990; Kaye and Mackintosh 1990). In contrast, rats given prenatal choline supplementation did not show less responding when the CS was extinguished in a new context. Instead, SUP rats extinguished at similar rates in both familiar and novel contexts.

These results are rather dramatic and can be interpreted in several ways. One simple explanation would be that SUP rats do not effectively process contextual cues and are thus insensitive to the differences between the contexts. However, that explanation is unlikely to be correct because of the data from the renewal phase of training. In this phase, both SUP and CON rats effectively used contextual retrieval cues to renew initially high levels of conditioned responding, whereas DEF rats did not. Thus, SUP rats are clearly capable of discriminating between contexts; these rats simply do not respond less (i.e., show a generalization dec-

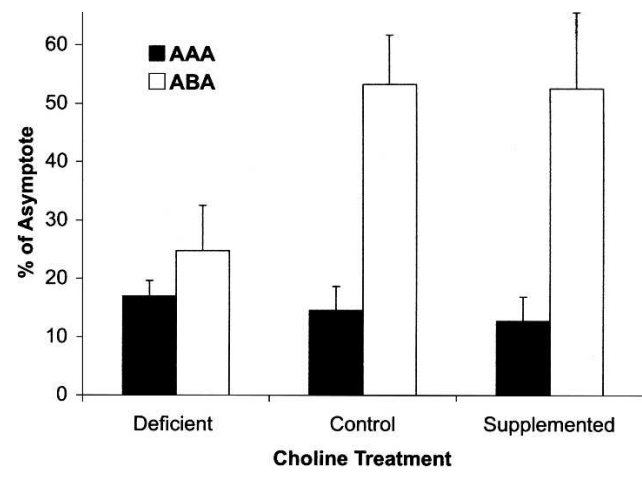

Figure 4. Conditioned responding of rats in a renewal test of appetitive Pavlovian conditioning as a function of prenatal choline availability. After extinction training, all rats were tested for responding to the noise CS in the context in which acquisition training had occurred. Rats in the AAA condition received acquisition, extinction, and renewal testing in the same physical context; rats in the ABA condition received extinction trials in a context different from that of acquisition training and renewal testing. Data shown are group means \pm SEM of the time spent in the food cup during CS presentations on the two final test sessions, expressed as a percentage of asymptotic responding. rement) when a CS is initially presented in a novel context. It should also be noted that the failure of the DEF rats to exhibit renewal (increased responding when the original context is restored) cannot be due to the fact that they are insensitive to differences in the contexts because the context switch during extinction produced a highly significant change in performance. Consequently, the double-dissociation of prenatal choline treatment and use of contextual cues as a function of extinction/ renewal conditions suggests that these conditioning procedures are mediated by different brain/behavioral processes.

\section{Experiment 3: Acquisition and retardation testing of Pavlovian conditioned inhibition}

The mean \pm SEM food-cup entry rates for all prenatal choline treatment groups during the initial phase of conditionedinhibition training are plotted as a function of three-session blocks in Figure 5. Responding is shown separately for reinforced noise-alone (+) trials and nonreinforced light/noise (-) trials for all three treatment groups. The rats in all treatment groups gradually learned to respond more on reinforced noise-alone $(+)$ trials than on nonreinforced light/noise (-) trials. Importantly, the rate of acquisition did not differ between any of the prenatal choline treatment groups. These claims are supported by a treatment $\times$ trial type $\times$ block ANOVA on the response rate data, which revealed reliable effects only for the main effect of trial type $\left(F_{(1,17)}=89.24 ; P<0.01\right)$ and the trial type $\times$ block interaction $\left(F_{(4,68)}=24.79 ; P<0.01\right)$. Neither the main effect of

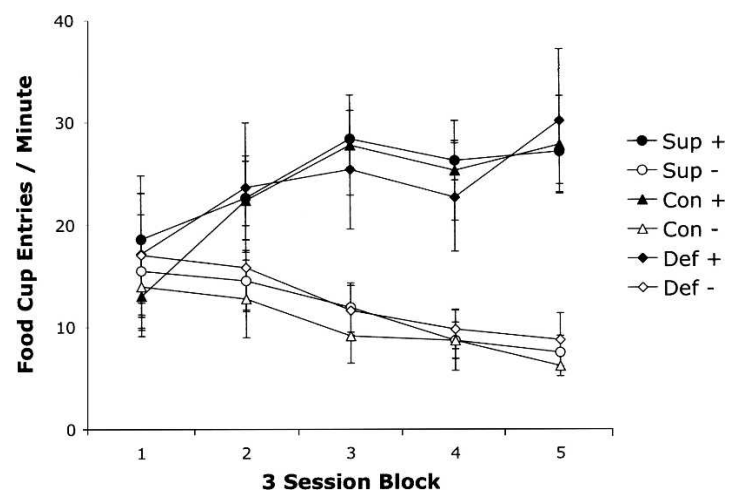

Figure 5. Acquisition of Pavlovian conditioned inhibition as a function of prenatal choline availability. Data represent group mean response rates \pm SEM of food-cup entry on reinforced [noise $\rightarrow$ food] trials (+, filled symbols) and nonreinforced [light/noise $\rightarrow$ no food] trials $(-$, open symbols) during Pavlovian conditioned inhibition training. SUP indicates supplemented group; CON, control group; and DEF, deficient group. 
treatment nor any other interactions reached statistical significance, $F s<1$. Planned comparisons showed further that none of the prenatal choline treatment groups differed in response rates either on the reinforced (+) or nonreinforced (-) trials either averaged across training or at asymptote, all $F \mathrm{~s}<1$.

Mean \pm SEM food-cup entry rates for all prenatal choline treatment groups during the retardation phase of training are shown in Figure 6 . All groups of rats rapidly acquired responding to the light CS. Responding reached asymptotic levels by the fourth daily session, and there were no differences in either the rates of acquisition or the asymptotic levels of conditioning among any of the treatment groups. These claims are supported by a treatment $\times$ session ANOVA, which revealed a significant main effect of session $\left(F_{(9,153)}=16.40 ; P<0.01\right)$, but no effect of treatment and no interactions between treatment and session, largest $F_{(18,153)}=1.03$.

The results of this experiment demonstrate that prenatal choline availability does not alter rats' ability to acquire a discrimination between reinforced (+) and unreinforced (-) trials or in the associated levels of conditioned inhibition as indicated in the retardation testing with the light CS.

\section{Discussion}

Prenatal nutrition sets the stage for brain function in later life, in part, through the process of "metabolic imprinting," which controls the rate of neurotransmitter synthesis, storage, release, and uptake (e.g., Blusztajn et al. 1998; Meck and Williams 2003; Wong-Goodrich et al. 2008b). Numerous research reports have described the importance of choline for the developing fetus and neonate (e.g., Zeisel 2000, 2004, 2005, 2006; McCann et al. 2006). This essential nutrient is involved in 1-carbon metabolism and is the precursor for many important compounds, including phospholipids, acetylcholine, and the methyl donor betaine. Dietary intake of choline by the pregnant mother and later by the infant directly affects brain development. In rodents, perinatal supplementation of choline enhances spatial and temporal cognition, changes that endure across the lifespan (Williams et al. 1998; Meck and Williams 2003; Meck et al. 2008). Conversely, choline deficiency during these sensitive periods results in altered memory function and associated cognitive deficits that also persist (Meck and Williams 1997c, 1999, 2003). Furthermore, recent studies suggest that perinatal choline supplementation can alleviate the cognitive effects of postnatal, human-third-semester pregnancy equivalent, alcohol exposure in rat offspring (e.g., Thomas et al. 2000, 2004, 2007), and ameliorate brain growth factor and sensorimotor deficiencies in mouse models of schizophrenia and Rett syndrome (e.g., Nag and Berger-Sweeney 2007;

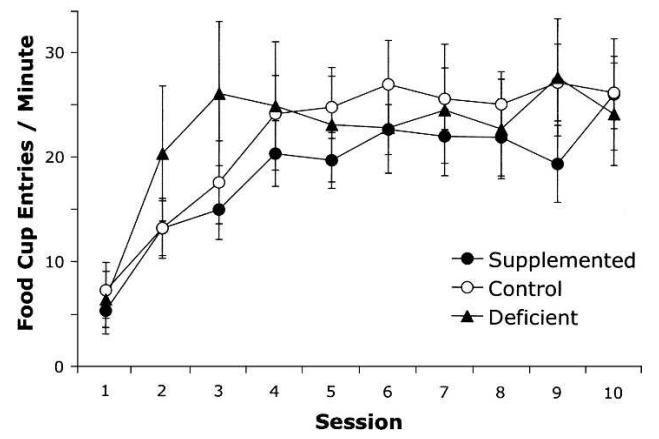

Figure 6. Retardation test of Pavlovian conditioned inhibition as a function of prenatal choline availability. Data represent group mean response rates \pm SEM of food-cup entry on reinforced [light $\rightarrow$ food] trials that followed Pavlovian conditioned inhibition training.
Stevens et al. 2008). The likely mechanism for these effects of choline involves an epigenetic change in DNA methylation, altered gene expression, and resulting changes in stem cell proliferation and differentiation (Zeisel 2006). Although the currently available animal data on prenatal choline availability and its long-term effects on hippocampal development and function are compelling (e.g., Albright et al. 2005), further studies are needed to determine whether humans could benefit in the same fashion from a prenatal choline supplemented diet (e.g., Zeisel and Blusztajn 1994; Zeisel, 2005; Signore et al. 2008).

Research dealing with the organizational effects of perinatal choline availability on adult cognitive performance has focused primarily upon spatial memory capacity as indexed by solution of radial-arm or water maze tasks as well as the content and precision of temporal memory for event durations in the supraseconds range (e.g., Meck and Williams 2003). In contrast, relatively little is known about the effects of prenatal choline manipulation on other types of learning and memory. For example, Tees (1999b) and Tees and Mohammadi (1999) reported that adult rats given perinatal choline supplementation showed enhanced performance on a nonspatial discrimination task. This task employed transverse patterning, which involves learning a very complex conditional discrimination. The studies reported here are the first to examine the effects of prenatal choline availability on basic associative learning processes in a manner similar to what has been done for interval timing (e.g., Meck et al. 1986; Meck 1987, 2002; Meck and Williams, 1997a,c; Cheng et al. 2006, 2008a,b; Cheng and Meck 2007; for a discussion of the role of temporal processing in learning and cognition, see Savastano and Miller 1998; Paule et al. 1999; Gallistel and Gibbon 2000; MacDonald and Meck 2004; Buhusi and Meck 2005, 2006; Meck and MacDonald 2007; Buhusi et al. 2008).

In the present study, the prenatal availability of choline did not affect the acquisition or extinction of simple conditioned excitation, or the acquisition or retardation of Pavlovian conditioned inhibition. However, prenatal choline availability did affect the contextual control of associative learning following both acquisition and extinction. SUP rats showed less dependency/ reliance on contextual cues following simple excitatory conditioning, as assessed by transfer to an alternate training context for extinction training. In contrast, DEF rats were impaired in their ability to use contexts as retrieval cues to renew responding after extinction, which is known to be dependent upon hippocampal function and related basal forebrain connections (Honey and Good 1993; Holland and Bouton 1999).

These results suggest that prenatal choline availability alters the manner in which the hippocampus processes the training context during Pavlovian conditioning, and are consistent with several modern accounts of associative learning. For example, Bouton (1991) has proposed that simple Pavlovian conditioning results in an associative structure that does not include connections with the training context. During extinction, however, an inhibitory association is established between the CS and US that is gated by the representation of the extinction context. According to this theory, renewal occurs because the inhibitory association learned during extinction is not expressed in the original training context. In the present experiment, the DEF rats' failure to show renewal may thus be due to the fact that they are relatively poor at processing the training context, and thus the inhibition learned in extinction generalizes to other contexts more than it does for SUP or CON rats. What is perhaps surprising is that the SUP rats show no loss of conditioned responding when undergoing extinction training in the novel context. This result can be explained by neural-network models of associative learning in which enhanced contextual processing in the hippocampus (as may be occurring in SUP rats) leads to a greater loss of 
associative strength for contextual cues during extinction than would be expected under moderate or impaired levels of contextual processing during initial acquisition, as observed in CON and DEF rats, respectively (e.g., Schmajuk and DiCarlo 1992; Schmajuk et al. 1998). As a consequence, enhanced contextual processing in combination with extinction of the "CS/context" complex would be expected to result in less reliance upon the training context for production of the CR and greater transfer of CS-US associations from one context to another. In other words, prenatal availability of choline may modify the contextprocessing efficacy of the septal-hippocampal pathway, with rats exposed to low levels of choline showing the least and rats exposed to high levels of choline showing the most efficient contextual processing. If this were true, it would explain the data described here. DEF rats show a loss of responding when placed in a novel context for extinction because they have failed to process the original context well enough to learn that it does not predict food delivery very effectively, i.e., that the CS is the only cue to which they need attend. In this scenario, the processing of the context by DEF rats is so inefficient that they are unable to use it as a discriminative retrieval cue for the initial conditioning phase when they are subsequently placed back in the familiar context for renewal testing. In contrast, SUP rats may process contextual cues very efficiently; thus they are easily able to use the contexts to renew their conditioned responding. The reason that SUP rats do not show a response decrement in the novel context during extinction is because they have processed the contextual cues in the initial conditioning phase and determined that they do not predict the US nearly as well as the noise CS.

A theoretical model of the developmental effects of prenatal choline availability on associative learning, based on Bouton's (1991, 1993) retrieval model of conditioning and extinction, is illustrated in Figure 7. Panel A (Fig. 7) depicts a model of conditioning processes that underlie simple Pavlovian conditioning, extinction, and contextual control of responding. Representations of both the CS and the context enter into associations with that of the US. Configural representations, established in a separate binding site, may also gain connections with the US representation. Figure 7, panel B shows how choline availability during early development may alter these systems to account for the current findings. Prenatal choline availability does not affect simple CS-US learning but alters contextual control of responding both during extinction and subsequent testing. This may be due to a singular effect on processing of contextual cues or by changes in multiple brain regions that perform specific associative functions. Further experimentation may support a distinction between these two possibilities, thus allowing us to determine whether the hippocampus contributes to configural learning by selectively enhancing cortical units representing stimulus conjunctions (e.g., Rudy and Sutherland 1995; Kennedy and Shapiro 2004).

The food-cup entry data reported in experiment 2 also address a long-running inconsistency in the Pavlovian conditioning literature. Although the loss of responding observed when the CON and DEF treatment groups first encountered the noise $\mathrm{CS}$ in context $\mathrm{B}$ is typical of the appetitive conditioning findings from some laboratories (e.g., Lovibond et al. 1984), other researchers consistently find no such loss in conditioned excitation with a context switch (e.g., Bouton and Swartzentruber 1986; Bouton 1991, 1993; Bouton and Ricker 1994). Additionally, recent studies report that lesions of the fimbria-fornix (Wilson et al. 1995) or the hippocampus (Frohardt et al. 2000) had no effect on conditioned excitation, inhibition, or renewal. Although the latter results are in direct conflict with the current results, the differences may be due to the nature of the behavioral task. Many of the studies that show a loss of excitation with a context switch
A

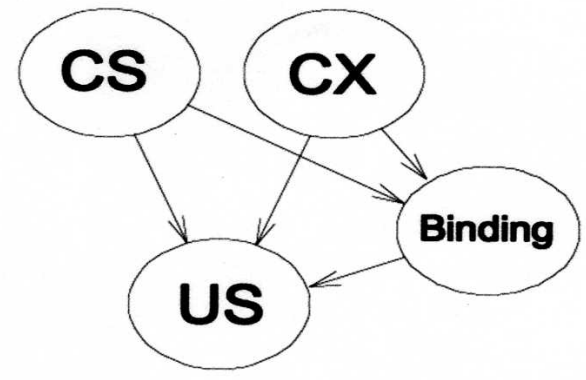

B

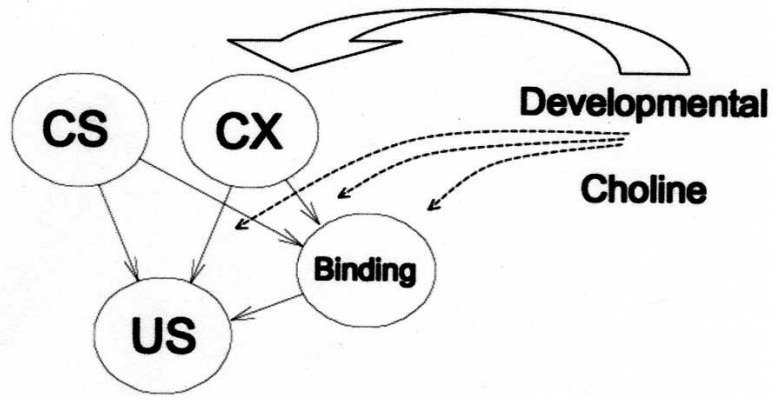

Figure 7. A neural-network model of the effects of variation in prenatal choline availability on Pavlovian conditioning processes. Panel $A$ depicts a model of Pavlovian conditioning, extinction, and contextual control of responding. Representations of both the CS and the context (CX) enter into associations with that of the US. Configural representations, comprised of combinations of multiple CSs and/or contextual cues, are established in a separate binding site and may also establish connections with the US representation. Panel $B$ shows how choline availability during neural development may alter these systems to account for the current data. Long-lasting alterations in neural circuits produced by prenatal choline levels do not affect simple CS-US learning but alter contextual control of responding both during extinction and subsequent testing. This may be due to a singular effect on the neural regions responsible for processing of contextual cues (indicated by the large, solid arrow) or by changes in multiple brain regions involved in establishing connections between contextual cues and either CSs or the US (indicated by the dashed arrows).

use appetitive-conditioning procedures, while all of the studies showing no loss involve aversive conditioning. In fact, one study included both a loss in responding in an appetitive design and no loss in an aversive design (Hall and Honey 1989). Honey and Good (1993) have also reported that selective hippocampal lesions abolish the contextual specificity of latent inhibition and conditioning in a variety of situations that is consistent with the current findings. Consequently, it is likely that different mechanisms are involved in learning appetitive and aversive tasks that involve different levels of arousal and/or stimulus salience (Martin-Soeich et al. 2007). The present series of experiments used appetitive-conditioning procedures, so it is perhaps not surprising that the $\mathrm{CON}$ rats in our study showed a loss of responding with a context switch during the extinction phase. Moreover, it may be that different brain regions are responsible for the phenomenon of renewal in aversive and appetitive situations. Therefore, the fact that hippocampal damage had no effect on renewal in an aversive design (Wilson et al. 1995; Frohardt et al. 2000) would not be incompatible with the present data. Even if the two 
effects are subserved by the same mechanisms, the results may not be at odds given the fact that DEF rats that failed to show renewal are not necessarily the same as hippocampally lesioned animals; rather, they likely have hippocampi that have developed in a qualitatively different manner from SUP and CON rats. Indeed, certain aspects of cholinergic metabolism in brain (specifically, acetylcholinesterase and high-affinity choline uptake) and behavior have been shown to be enhanced in DEF rats (e.g., Blusztajn et al. 1998; Cermak et al. 1998; Meck and Williams 1999, 2003; Wong-Goodrich et al. 2008b). Additionally, although hippocampal slices from DEF rats show increased thresholds for the induction of LTP (Pyapali et al. 1998; Jones III et al. 1999), it is not a trivial point that all slices showed LTP, i.e., DEF rats still have an active hippocampus, albeit with altered function.

In summary, the present series of experiments add to a growing body of findings showing that choline availability during ED 12-17, the time of origin of cholinergic neurons in the rat basal forebrain (Semba and Fibiger 1988) is crucial for the development of a number of cognitive abilities that are dependent, in part, on cholinergic input to and relational processing by the hippocampus (e.g., Dallal and Meck 1990; Halford et al. 1998; O'Reilly and Rudy 2001; Meck and Williams 2003; Dupret et al. 2008). It is important to note, however, that although prenatal choline availability has been shown to primarily affect the septum and hippocampus (e.g., Meck and Williams 2003; Mellott et al. 2007b; Meck et al. 2008), other brain regions such as the frontal cortex and striatum are also modified by these treatments and may contribute to some of the observed behavioral effects (e.g., Meck et al. 1989; Sandstrom et al. 2002; Mellott et al. 2007a; Nag et al. 2008).

\section{Materials and Methods}

\section{Experiment 1}

\section{Subjects}

Thirty naïve male Sprague-Dawley CD strain rats, the offspring of timed-pregnant dams obtained from Charles River Laboratories (Kingston, NY), served as subjects. All dams were obtained on ED 9 and fed AIN-76A purified synthetic diet containing $1.1 \mathrm{~g} / \mathrm{kg}$ choline chloride (Dyets) and water ad libitum. The manipulation of dietary choline took place from the evening of ED 11 through the morning of ED 18, after which all dams were fed a standard purified diet. During the treatment period, all dams received drinking water containing $50 \mathrm{mM}$ saccharin. Choline was supplemented (SUP) for one group of dams $(n=10)$ with the addition of $25 \mathrm{mM}$ choline chloride in the saccharin drinking water. A second group of dams $(n=10)$ received drinking water without choline and was switched to a purified diet completely deficient (DEF) of choline. The third group of dams $(n=10)$ served as a control $(\mathrm{CON})$ and was maintained on the standard purified diet (1.1 $\mathrm{g} / \mathrm{kg}$ choline chloride) and drinking water without added choline. Dams were housed individually in clear polycarbonate cages $(27.9 \times 27.9 \times 17.8 \mathrm{~cm})$ in a colony room maintained on a $12-12 \mathrm{~h}$, light-dark cycle.

Litters of 10 male pups, each containing rats from all dietary choline treatments (SUP, DEF, and CON), were cross-fostered at birth to untreated control dams. Pups were weaned at $24 \mathrm{~d}$ postnatal (PD 24) and housed five/cage at PD 30 and then two/cage on PD 45. These offspring received the standard AIN-76A diet described above and tap water ad libitum from weaning until PD 90. Throughout the remainder of the study, the rats were maintained at $85 \%$ of their ad libitum weights by limiting their access to food; water was still available continuously in the home cages. Thirty male rats from each of the dietary manipulations were selected for experiment 1 , and another 60 rats were used in experiment 2. Experimental training sessions were conducted on successive days during the light portion of the light-dark cycle. Training began when the rats were $\sim 100 \mathrm{~d}$ of age.

\section{Apparatus}

Ten identical conditioning chambers (model ENV-007, Med Associates) measuring $30.5 \times 24.1 \times 29.2 \mathrm{~cm}$ were used for this study. Each chamber was assigned to one of two sets that differed in olfactory, tactile, and spatial cues, thus providing two discriminable training contexts for the rats. In one set of chambers $(n=5)$, the front and back walls were aluminum and the side walls and top were clear polycarbonate. A food cup was recessed behind a $5 \times 5 \mathrm{~cm}$ aperture, centered along the front wall and $1.6 \mathrm{~cm}$ above the floor. An infrared photobeam-receptor circuit used to record food-cup entries was mounted inside the food cup, $1.3 \mathrm{~cm}$ behind the front wall, and $1.2 \mathrm{~cm}$ above the bottom of the wall aperture. A $2 \times 4.6-\mathrm{cm}$ response lever and a $100-\mathrm{mA}$ cue light, neither used in the present experiments, were centered 4.5 $\mathrm{cm}$ and $20 \mathrm{~cm}$, respectively, above the top of the food-cup aperture. A speaker that produced a $78-\mathrm{dB}(\mathrm{A})$ white noise was mounted behind the back wall, $3.5 \mathrm{~cm}$ from the left wall and 23 $\mathrm{cm}$ above the floor. This auditory cue was used as the primary CS in the experiments described below. The floor of the chamber consisted of $4.8-\mathrm{mm}$ stainless-steel rods, placed $1.6 \mathrm{~cm}$ apart. A drop tray $3.8 \mathrm{~cm}$ below the grid floor was lined with Fresh Step ${ }^{\circledR}$ brand cat litter (Clorox Pet Products).

In the second set of chambers $(n=5)$, the drop trays were filled with pine shavings, which provided a distinctively different odor cue. Also, a 20.5-cm-high aluminum insert was placed in each chamber, covering the left and back walls. In order to alter the geometry of the chamber, a 5-cm-high "shelf" projected $5 \mathrm{~cm}$ from the insert into the chamber and then down to the floor, running the entire width of the back wall. Finally, both the left and back walls of the insert, including both the horizontal and vertical surfaces of the shelf, were covered to a height of $12 \mathrm{~cm}$ by 60 -grit aluminum oxide sandpaper. This provided a substantial tactile difference between the two contexts. These two physical contexts, completely counterbalanced, served as the logical contexts $\mathrm{A}$ and $\mathrm{B}$ for the current experiments.

Each of the chambers was enclosed in a sound-attenuating shell. Background noise, $64 \mathrm{~dB}(\mathrm{~A})$, was provided by a ventilating fan on each shell. All chambers were connected to a single IBMcompatible computer, which controlled the presentation of all stimuli and the recording of responses.

\section{Behavioral procedures}

\section{Pavlovian conditioning procedures}

The rats were first trained to retrieve pellets from the food cup. All rats received two magazine training sessions, one in context A and one in context B. Each of the sessions was 32 min long and included eight trials consisting of the delivery of two $45-\mathrm{mg}$ Noyes food pellets, which served as the US for all the experiments described here. The intertrial interval (ITI) was fixed at $4 \mathrm{~min}$. One half of the rats received training in context A on session 1 and in context $\mathrm{B}$ on session 2; the sequence was reversed for the other half of the rats.

The rats were then given 10 daily conditioning sessions in context A. Each 32-min session contained eight trials in which the white noise CS was presented for $20 \mathrm{~s}$ and was immediately followed on each occasion by delivery of the US. ITIs were variable around a mean of 4 min with a square distribution, ranging from one-half to two times the mean interval. The rate of the rats' entry into the food-cup aperture ("nose-poking") was recorded during all CS presentations.

The 30 rats (10 from each choline treatment) were then given extinction training with the white-noise CS. These rats received four daily sessions in which the noise was presented eight times in the absence of food. For all rats, these sessions were conducted in the same physical training context in which they had received initial excitatory conditioning. The sessions were similar to those of the excitatory conditioning sessions, except that CS presentations were not followed by the delivery of food pellets. ITIs during these sessions were fixed at $4 \mathrm{~min}$. 


\section{Data collection and analysis}

The behavior of interest was the conditioned approach to the food cup, measured by the interruption of the photobeam relay across the food-cup aperture. Two response measures can be obtained: (1) the rate of food-cup entries during the CS, which is the most sensitive measure for short CS durations (e.g., $5 \mathrm{~s}$ ), and (2) the percentage of time spent in the food cup during the second half of the CS, which is most sensitive for longer CS durations (e.g., 20 s).

In addition, food-cup entries during extinction and subsequent test sessions were transformed into a percentage of each rat's asymptotic level of responding (the mean of the last four sessions of conditioning). This procedure controlled for differences in initial conditioning levels and provided a more accurate comparison of performance during nonreinforced extinction and test sessions. Main effects and interactions between choline conditions and behavioral training variables were subjected to ANOVA procedures. Planned comparisons were evaluated using separate contrasts. A $P<0.05$ criterion of statistical significance was adopted for all comparisons.

\section{Experiment 2}

\section{Subjects and apparatus}

The subjects were 60 male naïve rats derived from the litters described in experiment 1 , evenly divided among the SUP, DEF, and CON prenatal choline treatments. These rats were housed and maintained as described for experiment 1 , and the apparatus was the same.

\section{Behavioral procedures}

\section{Pavlovian conditioning procedures}

The rats were first trained to retrieve pellets from the food cup. All rats received two magazine training sessions, one in context $\mathrm{A}$ and one in context B. Each of the sessions was 32 min long and included eight trials consisting of the delivery of two $45-\mathrm{mg}$ Noyes food pellets, which served as the US for all the experiments described here. The ITI was fixed at $4 \mathrm{~min}$. One half of the rats received training in context $A$ on session 1 and in context $B$ on session 2; the sequence was reversed for the other half of the rats.

All of the rats were then given 10 daily conditioning sessions in context A. Each 32-min session contained eight trials in which the white noise CS was presented for $20 \mathrm{~s}$ and was immediately followed on each occasion by delivery of the US. ITIs were variable around a mean of 4 min with a square distribution, ranging from one-half to two times the mean interval. The rate of the rats' entry into the food-cup aperture (nose-poking) was recorded during all CS presentations. The rats then received four daily sessions of extinction training with the noise CS, identical to that described in experiment 1 . All rats received tight presentations of the noise CS for $20 \mathrm{~s}$ in the absence of food reinforcement. Each session last for $32 \mathrm{~min}$, and the ITI was fixed at $4 \mathrm{~min}$. Half of the rats from each choline treatment $(n ' s=10)$ were trained with the $\mathrm{ABA}$ condition, and the remaining rats from each treatment were trained on the AAA condition. Rats in AAA condition received the extinction trials in the same physical training context in which they had received initial excitatory conditioning (i.e., context A). Rats trained in the ABA condition received extinction training in the alternate training context (i.e., context B). On the two sessions following the last extinction session, all rats were placed in context A for renewal test sessions. The renewal test sessions were identical to the extinction sessions described above and were conducted in context A for all rats.

\section{Experiment 3}

\section{Subjects and apparatus}

The subjects were seven SUP, seven DEF, and six CON male rats bred and raised in the same manner as those used in experiments 1 and 2. Half of the rats in each treatment group were experimentally naïve. The other half had received prior simple Pavlov- ian excitation training with the noise stimulus, but had not received presentation of the light CS. Subjects were $\sim 160 \mathrm{~d}$ of age when the study began. The apparatus was the same as that used in the previous experiments; however, the two contexts did not serve as discriminative cues in this study.

\section{Behavioral procedures}

\section{Pavlovian-conditioning procedures}

The rats were first trained to retrieve food pellets from the food cup as in experiment 1 . Rats were then trained once/day for $15 \mathrm{~d}$. In each daily $64 \mathrm{~min}$ session, a 5-s white noise CS was presented 16 times. On four presentations each day, the noise was immediately followed by delivery of the US (food pellet). On the other 12 presentations of the noise, a light CS was simultaneously presented; no food pellets were delivered on these compound light/ noise trials. The rats were then given 10 daily 64 -min training sessions in which only the light CS was presented (retardation test). In each daily session, rats received 16 presentations of the light, followed immediately by the US (food pellet). In both phases of this experiment, ITIs were variable with a mean of 4 min having a square distribution, ranging from one-half to two times the mean interval. As explained in the Materials and Methods section, the rate of nose-poking in the food cup was used as the behavioral measure of interest. Food-cup entry rates were recorded during all CS presentations throughout the experiment.

Initial analysis of the food-cup entry data from both phases of the study showed that prior experimental history did not interact with any of the variables of interest. Naïve rats responded less to the noise than the experienced rats at the outset of the study, but this affected neither the acquisition of the initial discrimination nor subsequent retardation testing and moreover did not differ between choline treatment groups. Prior experience was thus excluded as a variable in the final statistical analyses presented here in order to enhance statistical power.

\section{Acknowledgments}

This research was supported by grant AG09525 from the National Institute of Aging. We thank Jean-Paul Paskalis for assistance with testing the animals and Catalin Buhusi and Eric Mohler for their thoughtful comments on the manuscript.

\section{References}

Albright, C.D., Mar, M.H., Craciunescu, C.N., Song, J., and Zeisel, S.H. 2005. Maternal dietary choline availability alters the balance of netrin-1 and DCC neuronal migration proteins in fetal mouse brain hippocampus. Brain Res. Dev. Brain Res. 159: 149-154.

Blusztajn, J.K. 1998. Choline, a vital amine. Science 281: 794-795.

Blusztajn, J.K. and Wurtman, R.J. 1983. Choline and cholinergic neurons. Science 221: 614-620.

Blusztajn, J.K., Cermak, J.M., Holler, T., and Jackson, D.A. 1998. Imprinting of hippocampal metabolism of choline by its availability during gestation: Implications for cholinergic neurotransmission. $J$. Physiol. 92: 199-203.

Bouton, M.E. 1991. Context and retrieval in extinction and in other examples of interference in simple associative learning. In Current topics in animal learning: Brain, emotion, and cognition (eds. L. Dachowski and C.F. Flaherty), pp. 25-53. Lawrence Erlbaum, Hillsdale, NJ.

Bouton, M.E. 1993. Context, time, and memory retrieval in the interference paradigms of Pavlovian learning. Psych. Bull. 114: 80-99.

Bouton, M.E. and Bolles, R.C. 1979. Contextual control of the extinction of conditioned fear. Learn. Motiv. 10: 445-466.

Bouton, M.E. and Ricker, S.T. 1994. Renewal of extinguished responding in a second context. Anim. Learn. Behav. 22: 317-324.

Bouton, M.E. and Swartzentruber, D. 1986. Analysis of the associative and occasion-setting properties of contexts participating in a Pavlovian discrimination. J. Exp. Psych. Anim. Behav. Process. 12: 333-350.

Brandner, C. 2002. Perinatal choline treatment modifies the effects of a visuo-spatial attractive cue upon spatial memory in naïve adult rats. Brain Res. 928: 85-95.

Buhusi, C.V. and Meck, W.H. 2005. What makes us tick? Functional and neural mechanisms of interval timing. Nat. Rev. Neurosci. 6: 755-765.

Buhusi, C.V. and Meck, W.H. 2006. Interval timing with gaps and 
distracters: Evaluation of the ambiguity, switch, and time-sharing hypotheses. J. Exp. Psychol. Anim. Behav. Process. 32: 329-338.

Buhusi, C.V., Lamoureux, J.A., and Meck, W.H. 2008. Prenatal choline supplementation increases sensitivity to contextual processing of temporal information. Brain Res. 1237: 204-213.

Cermak, J.M., Holler, T., Jackson, D.A., and Blusztajn, J.K. 1998. Prenatal availability of choline modifies the development of the hippocampal cholinergic system. FASEB J. 12: 349-359.

Cermak, J.M., Blusztajn, J.K., Meck, W.H., Williams, C.L., Fitzgerald, C.M., Rosene, D.L., and Loy, R. 1999. Prenatal availability of choline alters the development of acetylcholinesterase in rat hippocampus. Dev. Neurosci. 21: 94-104.

Chan, K.H., Morell, J.R., Jarrard, L.E., and Davidson, T.L. 2001. Reconsideration of the role of the hippocampus in learned inhibition. Behav. Brain Res. 119: 111-130.

Cheng, R.K. and Meck, W.H. 2007. Prenatal choline supplementation increases sensitivity to time by reducing non-scalar sources of variance in adult temporal processing. Brain Res. 1186: 242-254.

Cheng, R.K., Meck, W.H., and Williams, C.L. 2006. $\alpha 7$ nicotinic acetylcholine receptors and temporal memory: Synergistic effects of combining prenatal choline and nicotine on reinforcement-induced resetting of an interval clock. Learn. Mem. 13: 127-134.

Cheng, R.K., MacDonald, C.J., Williams, C.L., and Meck, W.H. 2008a Prenatal choline supplementation alters the timing, emotion, and memory performance (TEMP) of adult male and female rats as indexed by differential reinforcement of low-rate schedule behavior. Learn. Mem. 15: 153-162.

Cheng, R.K., Scott, A.C., Williams, C.L., and Meck, W.H. 2008b. Modality-specificity of duration discrimination in vision and audition as a function of prenatal choline availability. Brain Res. 1237: $167-175$.

Dallal, N.L. and Meck, W.H. 1990. Hierarchical structures: Chunking by food type facilitates spatial memory. J. Exp. Psychol. Anim. Behav. Process. 16: 69-84.

Dupret, D., Revest, J.-M., Koehl, M., Ichas, F., De Giorgi, F., Costet, P., Abrous, D.N., and Piazza, P.V. 2008. Spatial relational memory requires hippocampal adult neurogenesis. PLoS One 3: e1959. doi: 10.1371/journal.pone.0001959.

Frohardt, R.J., Guarraci, F.A., and Bouton, M.E. 2000. The effects of neurotoxic hippocampal lesions on two effects of context after fear extinction. Behav. Neurosci. 114: 227-240.

Gallistel, C.R. and Gibbon, J. 2000. Time, rate, and conditioning. Psychol. Rev. 107: 289-344.

Glenn, M.J., Gibson, E.M., Kirby, E.D., Mellott, T.J., Blusztajn, J.K., and Williams, C.L. 2007. Prenatal choline availability modulates hippocampal neurogenesis and neurogenic responses to enriching experiences in adult female rats. Eur. J. Neurosci. 25: 2473-2482.

Good, M., de Hoz, L., and Morris, R.G.M. 1997. Contingent versus incidental context processing during conditioning: Dissociation after excitotoxic hippocampal plus dentate gyrus lesions. Hippocampus 8: 147-159.

Green, J.T. and Woodruff-Pak, D.S. 2000. Eyeblink classical conditioning: Hippocampal formation is for neutral stimulus associations as cerebellum is for association-response. Psychol. Bull. 126: $138-158$.

Halford, G.S., Wilson, W.H., and Phillips, S. 1998. Processing capacity defined by relational complexity: Implications for comparative, developmental, and cognitive psychology. Behav. Brain Sci. 21: 803-865.

Hall, G. and Honey, R.C. 1989. Contextual effects in conditioning, latent inhibition, and habituation: Associative and retrieval functions of contextual cues. J. Exp. Psychol. Anim. Behav. Process. 15: 232-241.

Hall, G. and Honey, R.C. 1990. Context-specific conditioning in the conditioned-emotional- response procedure. J. Exp. Psychol. Anim. Behav. Process. 16: 271-278.

Holland, P.C. and Bouton, M.E. 1999. Hippocampus and context in classical conditioning. Curr. Opin. Neurobiol. 9: 195-202.

Holland, P.C., Lamoureux, J.A., Han, J.S., and Gallagher, M.H. 1999. Hippocampal lesions interfere with Pavlovian negative occasion setting. Hippocampus 9: 143-157.

Holler, T., Cermak, J.M., and Blusztajn, J.K. 1996. Dietary choline supplementation in pregnant rats increases hippocampal PLD activity of the offspring. FASEB J. 10: 1653-1659.

Holmes, G.L., Yang, Y., Liu, Z., Cermak, J.M., Sarkisian, M.R., Stafstrom, C.E., Neill, J.C., and Blusztajn, J.K. 2002. Seizure-induced memory impairment is reduced by choline supplementation before or after status epilepticus. Epilepsy Res. 48: 3-13.

Honey, R.C. and Good, M. 1993. Selective hippocampal lesion abolish the contextual specificity of latent inhibition and conditioning. Behav. Neurosci. 107: 23-33.

Honey, R.C., Willis, A., and Hall, G. 1990. Context specificity in pigeon autoshaping. Learn. Motiv. 21: 125-136.

Jarrard, L.E. and Davidson, T.L. 1990. Acquisition of concurrent conditional discriminations in rats with ibotenate lesions of hippocampus and of subiculum. Psychobiology 18: 68-73.

Jarrard, L.E. and Davidson, T.L. 1991. On the hippocampus and learned conditional responding: Effects of aspiration versus ibotenate lesions. Hippocampus 1: 103-113.

Jones III, J.P., Meck, W.H., Williams, C.L., Wilson, W.A., and Swartzwelder, S.H. 1999. Choline availability to the developing rat fetus alters adult hippocampal long-term potentiation. Brain Res. Dev. Brain Res. 118: 159-167.

Kaye, H. and Mackintosh, N.J. 1990. A change of context can enhance performance of an aversive but not of an appetitive conditioned response. Quart. J. Exp. Psychol. Comp. Physiol. Psychol. 42: 113-134.

Kennedy, P.J. and Shapiro, M.L. 2004. Retrieving memories via internal context requires the hippocampus. J. Neurosci. 24: 6979-6985.

Lovibond, P.F., Preston, G.C., and Mackintosh, N.J. 1984. Context specificity of conditioning, extinction, and latent inhibition. J. Exp. Psychol. Anim. Behav. Process. 10: 360-375.

MacDonald, C.J. and Meck, W.H. 2004. Systems-level integration of interval timing and reaction time. Neurosci. Biobehav. Rev. 28: 747-769.

Martin-Soeich, C., Linthicum, J., and Ernst, M. 2007. Appetitive conditioning: Neural bases and implications for psychopathology. Neurosci. Biobehav. Rev. 31: 426-440.

McCann, J.C., Hudes, M., and Ames, B.N. 2006. An overview of evidence for a causal relationship between dietary availability of choline during development and cognitive function in offspring. Neurosci. Biobehav. Rev. 30: 696-712.

McKeon-O'Malley, C., Siwek, D., Lamoureux, J.A., Williams, C.L., and Kowall, N.W. 2003. Prenatal choline deficiency decrease the cross-sectional area of cholinergic neurons in the medial septal nucleus. Brain Res. 977: 278-283.

Meck, W.H. 1987. Vasopressin metabolite neuropeptide facilitates simultaneous temporal processing. Behav. Brain Res. 23: 147-157.

Meck, W.H. 1988. Hippocampal function is required for feedback control of an internal clock's criterion. Behav. Neurosci. 102: 54-60.

Meck, W.H. 2002. Choline uptake in the frontal cortex is proportional to the absolute error of a temporal memory translation constant in mature and aged rats. Learn. Motiv. 33: 88-104.

Meck, W.H. and MacDonald, C.J. 2007. Amygdala inactivation reverses fear's ability to impair divided attention and make time still. Behav. Neurosci. 121: 707-720.

Meck, W.H. and Williams, C.L. 1997a. Characterization of the facilitative effects of perinatal choline supplementation on timing and temporal memory. Neuroreport 8: 2831-2835.

Meck, W.H. and Williams, C.L. 1997b. Perinatal choline supplementation increases the threshold for chunking in spatial memory. Neuroreport 8: 3053-3059.

Meck, W.H. and Williams, C.L. 1997c. Simultaneous temporal processing is sensitive to prenatal choline availability in mature and aged rats. Neuroreport 8: $3045-3051$.

Meck, W.H. and Williams, C.L. 1999. Choline supplementation during prenatal development reduces proactive interference in spatial memory. Brain Res. Dev. Brain Res. 118: 51-59.

Meck, W.H. and Williams, C.L. 2003. Metabolic imprinting of choline by its availability during gestation: Implications for memory and attentional processing across the lifespan. Neurosci. Biobehav. Rev. 27: 385-399.

Meck, W.H., Church, R.M., and Wenk, G.L. 1986. Arginine vasopressin inoculates against age-related increases in sodium-dependent high affinity choline uptake and discrepancies in the content of temporal memory. Eur. J. Pharmacol. 130: 327-331.

Meck, W.H., Smith, R.A., and Williams, C.L. 1988. Pre- and postnatal choline supplementation produces long-term facilitation of spatial memory. Dev. Psychobiol. 21: 339-353.

Meck, W.H., Smith, R.A., and Williams, C.L. 1989. Organizational changes in cholinergic activity and enhanced visuospatial memory as a function of choline administered prenatally or postnatally or both. Behav. Neurosci. 103: 1234-1241.

Meck, W.H., Williams, C.L., Cermak, J.M., and Blusztain, J.K. 2008 Developmental periods of choline sensitivity provide an ontogenetic mechanism for regulating memory capacity and age-related dementia. Front. Integr. Neurosci. 1: 7. doi: 10.3389/neuro.07.007.2007.

Mellott, T.J., Williams, C.L., Meck, W.H., and Blusztajn, J.K. 2004. Prenatal choline supplementation advances hippocampal development and enhances MAPK and CREB activation. FASEB J. 18: 545-547.

Mellott, T.J., Follettie, M.T., Diesl, V., Hill, A.A., Lopez-Coviella, I., and Blusztain, J.K. 2007a. Prenatal choline availability modulates hippocampal and cerebral gene expression. FASEB J. 21: 1311-1323. 
Mellott, T.J., Kowall, N.W., Lopez-Coviella, I., and Blusztajn, J.K. 2007b. Prenatal choline deficiency increases choline transporter expression in the septum and hippocampus during postnatal development and in adulthood in rats. Brain Res. 1151: $1-11$.

Mohler, E.G., Meck, W.H., and Williams, C.L. 2001. Sustained attention in adult mice is modulated by prenatal choline availability. Int. J. Comp. Psychol. 14: 136-150.

Montoya, D.A.C., White, A.M., Williams, C.L., Blusztajn, J.K., Meck, W.H., and Swartzwelder, H.S. 2000. Prenatal choline exposure alters hippocampal responsiveness to cholinergic stimulation in adulthood. Brain Res. Dev. Brain Res. 123: 25-32.

Nafee, T.M., Farrell, W.E., Carroll, W.D., Fryer, A.A., and Ismail, K.M.K. 2008. Epigenetic control of fetal gene expression. BJOG 115: $158-168$.

Nag, N. and Berger-Sweeney, J.E. 2007. Postnatal dietary choline supplementation alters behavior in a mouse model of Rhett syndrome. Neurobiol. Dis. 26: 473-480.

Nag, N., Mellott, T.J., and Berger-Sweeney, J.E. 2008. Effects of postnatal dietary choline supplementations on motor regional brain volume and growth factor expression in a mouse model of Rett syndrome. Brain Res. 1237: 101-109.

O'Reilly, R.C. and Rudy, J.W. 2001. Conjunctive representations in learning and memory: Principles of cortical and hippocampal function. Psychol. Rev. 108: 311-345.

Paule, M.G., Meck, W.H., McMillan, D.E., Bateson, M., Popke, E.J., Chelonis, J.J., and Hinton, S.C. 1999. The use of timing behaviors in animals and humans to detect drug and/or toxicant effects. Neurotoxicol. Teratol. 21: 491-502.

Pavlov, I.P. 1927. Conditioned reflexes. Oxford, London, UK.

Pyapali, G.K., Turner, D.A., Williams, C.L., Meck, W.H., and Swartzwelder, H.S. 1998. Prenatal dietary choline supplementation decreases the threshold for induction of long-term potentiation in young adult rats. J. Neurophysiol. 79: 1790-1796.

Rescorla, R.A. 1969. Pavlovian conditioned inhibition. Psychol. Bull. 72: 77-94.

Ross, R.T., Orr, W.B., Holland, P.C., and Berger, T.W. 1984. Hippocampectomy disrupts acquisition and retention of learned conditional responding. Behav. Neurosci. 98: 211-225.

Rudy, J.W. and Sutherland, R.J. 1995. Configural association theory and the hippocampal formation: An appraisal and reconfiguration. Hippocampus 5: 375-389.

Sandstrom, N.J., Loy, R., and Williams, C.L. 2002. Prenatal choline supplementation increases NGF levels in the hippocampus and frontal cortex of young and adult rats. Brain Res. 947: 9-16.

Savastano, H.I. and Miller, R.R. 1998. Time as content in Pavlovian conditioning. Behav. Processes 44: 147-162.

Semba, K. and Fibiger, H.C. 1988. Time of origin of cholinergic neurons in the rat basal forebrain. J. Comp. Neurol. 269: 87-95.

Schmajuk, N.A. and Buhusi, C.V. 1997. Stimulus configuration, occasion setting, and the hippocampus. Behav. Neurosci. 111: 235-258.

Schmajuk, N.A. and DiCarlo, J.J. 1992. Stimulus configuration, classical conditioning, and hippocampal function. Psychol. Rev. 99: 268-305.

Schmajuk, N.A., Lamoureux, J.A., and Holland, P.C. 1998. Occasion setting: A neural network approach. Psychol. Rev. 105: 3-32.

Signore, C., Ueland, P.M., Troendle, J., and Mills, J.L. 2008. Choline concentrations in human maternal and cord blood and intelligence at 5 y of age. Am. J. Clin. Nutr. 87: 896-902.

Solomon, P.R. 1977. Role of the hippocampus in blocking and conditioned inhibition of the rabbit's nictitating membrane response. J. Comp. Physiol. Psychol. 91: 407-417.

Stevens, K.E., Adams, C.E., Yonchek, J., Hickel, C., Danielson, J., and
Kisley, M.A. 2008. Permanent improvement in deficient sensory inhibition in DBA/2 mice with increased perinatal choline. Psychopharmacology 198: 413-420.

Tees, R.C. 1999a. The influences of rearing environment and neonatal choline dietary supplementation on spatial learning and memory in adult rats. Behav. Brain Res. 105: $173-188$.

Tees, R.C. 1999b. The influences of sex, rearing environment, and neonatal choline dietary supplementation on spatial and nonspatial learning and memory in adult rats. Dev. Psychobiol. 35: 328-342.

Tees, R.C. and Mohammadi, E. 1999. The effects of neonatal choline dietary supplementation on adult spatial and configural learning and memory in rats. Dev. Psychobiol. 35: 226-240.

Thomas, J.D., La Fiette, M.H., Quinn, V.R., and Riley, E.P. 2000. Neonatal choline supplementation ameliorates the effects of prenatal alcohol exposure on a discrimination learning task in rats. Neurotoxicol. Teratol. 22: 703-711.

Thomas, J.D., Garrison, M., and O’Neill, T.M. 2004. Perintala choline supplementation attenuates behavioral alterations associated with neonatal alcohol exposure in rats. Neurotoxicol. Teratol. 26: 35-45.

Thomas, J.D., Biane, J.S., O'Bryan, K.A., O'Neill, T.M., and Dominguez, H.D. 2007. Choline supplementation following third-trimester-equivalent alcohol exposure attenuates behavioral alterations in rats. Behav. Neurosci. 121: 120-130.

Wagner, A.F. and Hunt, P.S. 2006. Impaired trace fear conditioning following neonatal ethanol: Reversal by choline. Behav. Neurosci. 120: $482-487$

Waterland, R.A. and Michels, K.B. 2007. Epigenetic epidemiology of the developmental origins hypothesis. Annu. Rev. Nutr. 27: 363-388.

Williams, C.L., Meck, W.H., Heyer, D.D., and Loy, R. 1998. Hypertrophy of basal forebrain neurons and enhanced visuospatial memory in perinatally choline-supplemented rats. Brain Res. 794: 225-238.

Wilson, A., Brooks, D.C., and Bouton, M.E. 1995. The role of the rat hippocampal system in several effects of context in extinction. Behav. Neurosci. 109: 828-836.

Wong-Goodrich, S.J.E., Glenn, M.J., Mellott, T.J., Blusztajn, J.K., Meck, W.H., and Williams, C.L. 2008a. Spatial memory and hippocampal plasticity are differentially sensitive to the availability of choline in adulthood as a function of its supply in utero. Brain Res. 1237: $153-166$.

Wong-Goodrich, S.J.E., Mellott, T.J., Glenn, M.J., Blusztajn, J.K., and Williams, C.L. 2008b. Prenatal choline supplementation attenuates neuropathological response to status epilepticus in the adult rat hippocampus. Neurobiol. Dis. 30: 255-269.

Yang, Y., Cermak, J.M., Tandon, P., Sarkisian, M.R., Stafstrom, C.E., Neill, J.C., Blusztajn, J.K., and Holmes, G.L. 2000. Protective effects of prenatal choline supplementation on seizure-induced memory impairment. J. Neurosci. 20: 1-6.

Zeisel, S.H. 2000. Choline: Needed for normal development of memory. J. Am. Coll. Nutr. 19: 528S-531S.

Zeisel, S.H. 2004. Nutritional importance of choline for brain development. J. Am. Coll. Nutr. 23: 621S-626S.

Zeisel, S.H. 2005. Choline, homocysteine, and pregnancy. Am. J. Clin. Nutr. 82: 719-720.

Zeisel, S.H. 2006. The fetal origins of memory: The role of dietary choline in optimal brain development. J. Pediatr. 149: S131-S136.

Zeisel, S.H. and Blusztajn, J.K. 1994. Choline and human nutrition. Annu. Rev. Nutr. 14: 269-296.

Received May 1, 2008; accepted in revised form September 21, 2008. 


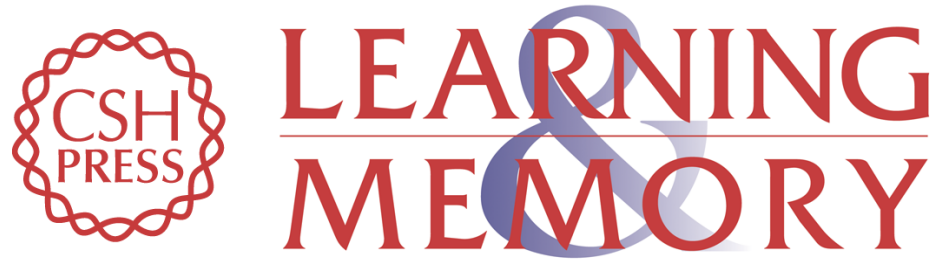

\section{Prenatal choline availability alters the context sensitivity of Pavlovian conditioning in adult rats}

Jeffrey A. Lamoureux, Warren H. Meck and Christina L. Williams

Learn. Mem. 2008, 15:

Access the most recent version at doi:10.1101//m.1058708

References This article cites 99 articles, 8 of which can be accessed free at:

http://learnmem.cshlp.org/content/15/12/866.full.html\#ref-list-1

License

Email Alerting Receive free email alerts when new articles cite this article - sign up in the box at the Service top right corner of the article or click here. 